\title{
High stability of trehalose/maltose binding protein from Thermococcus litoralis makes it a good candidate as a sensitive element in biosensor systems for sugar control
}

\author{
Olga I. Povarova ${ }^{\text {a,* }}$, Olga V. Stepanenko ${ }^{\text {a }}$, Anna I. Sulatskaya ${ }^{a}$, Irina M. Kuznetsova ${ }^{\text {a }}$, \\ Konstantin K. Turoverov ${ }^{\mathrm{a}}$, Maria Staiano ${ }^{\mathrm{b}}$, Annalisa Vitale ${ }^{\mathrm{b}}$ and Sabato D'Auria ${ }^{\mathrm{b}}$ \\ ${ }^{a}$ Institute of Cytology of the Russian Academy of Sciences, Saint-Petersburg, Russia \\ ${ }^{\mathrm{b}}$ Institute of Protein Biochemistry CNR, Napoli, Italy
}

\begin{abstract}
Fluorescence and circular dichroism in far-UV region were used to study the stability of trehalose/maltose binding protein (TMBP) from hyper thermophilic archaeon Thermococcus litoralis and its complex with glucose (TMBP/Glc). The evaluation of difference between free energy of native and unfolded state for TMBP and TMBP/Glc showed that both of them are several times higher than that of proteins from mesophilic organisms. Due to the high stability and innate ability to bind glucose this protein is a good candidate as a sensitive element in biosensor systems for sugar control.
\end{abstract}

Keywords: Fluorescence, circular dichroism, hyperthermophilic organism, protein stability

\section{Introduction}

Highly stable structure of proteins from extremophilic organisms makes them an interesting object for fundamental investigations of protein folding and structural stability study. This property makes these proteins a good candidate as a sensitive element in biosensor systems. Trehalose/Maltose Binding Protein (TMBP) is a component of the trehalose and maltose uptake system of hyperthermophilic organism Thermococcus litoralis [10]. TMBP consists of two domains connected by a hinge which is formed by two antiparallel $\beta$-sheets and one $\alpha$-helix [2]. The substrate binding site is located in the cleft between domains. In vivo the native ligands of TMBP are trehalose and maltose, but TMBP can bind also glucose [3]. TMBP can be used as a sensitive element of non-consuming biosensor for monitoring of the glucose level in blood. The goal of this work was a detailed examination of physical-chemical properties and stability of TMBP and its complex with D-glucose (TMBP/Glc). To this end the conformational changes of the protein and its complex with D-glucose induced by guanidine hydrochloride ( $\mathrm{GdnHCl})$ and heating were studied by intrinsic fluorescence and far-UV circular dichroism (CD).

\footnotetext{
* Corresponding author: Dr. Olga I. Povarova, Scientific Associate of the Laboratory of Structural Dynamics, Stability and Protein Folding, Institute of Cytology RAS, Saint-Petersburg, Russia. Tel.: +7 812297 19 57; Fax: +7 81229735 41; E-mail: olp@mail.cytspb.rssi.ru.
} 


\section{Materials and methods}

\subsection{Sample handling}

Recombinant TMBP was purified as described elsewhere [1,4]. The protein concentration was determined by spectrophotometry (EPS-3T, Hitachi, Japan). The molar extinction constant for TMBP was taken as E280 $=1.94(\mathrm{mg} / \mathrm{ml} \cdot \mathrm{cm})^{-1}$. This value was calculated based on fact that TMBP contain 22 tyrosines and 12 tryptophans [2] and molecular weight of TMBP is $48 \mathrm{kDa}$ [3]. The protein concentration was $0.3 \mathrm{mg} / \mathrm{ml}$. For complex formation, $10^{-2} \mathrm{~mol} / \mathrm{l} \mathrm{Glc}$ was added. In all experiments $20 \mathrm{mM}$ Tris- $\mathrm{HCl}$ buffer ( $\mathrm{pH}$ 7.5) was used. $\mathrm{GdnHCl}$ was used without additional purification. The $\mathrm{GdnHCl}$ concentration was determined by refraction index with an Abbe refractometer (LOMO, Russia). Solutions of protein with $\mathrm{GdnHCl}$ were incubated during $24 \mathrm{~h}$ at room temperature. All chemicals used in the present study were from Sigma (USA).

\subsection{Fluorescence measurements}

Fluorescence experiments were performed with steady-state spectrofluorimeter [7]. Fluorescence was excited at $297 \mathrm{~nm}$. The parameter $A\left(=I_{320} / I_{365}\right.$, where $I_{320}$ and $I_{365}$ are the fluorescence intensity at 320 and $365 \mathrm{~nm}$, respectively) was used to characterize fluorescence spectra position [8]. The fluorescence spectra and the values of parameter $A$ were both corrected by the instrument spectral sensitivity. In case of $\mathrm{GdnHCl}$-induced denaturation of TMBP and TMBP/Glc, experiments were carried out at $23^{\circ} \mathrm{C}$. The equilibrium dependences of fluorescence intensity at the fixed wavelength of registration upon $\mathrm{GdnHCl}$ additions for TMBP and complex TMBP/Glc were used to evaluate the free energy differences of GGBP and GGBP/Glc in native and unfolded states $\Delta G \mathrm{~N}-\mathrm{U}(0)$. The calculations were performed according to the standard scheme [6].

\subsection{Circular dichroism spectroscopy}

We used the spectropolarimeter J-810 (Jasco, Japan) equipped with the temperature-controlled liquid system Julabo F-25 (Julabo USA Inc., USA). Cuvettes with a path length of $0.1 \mathrm{~cm}$ (Helma, Germany) were used. The photomultiplier voltage never exceeded $600 \mathrm{~V}$ in the spectral regions that were measured. Each spectrum was averaged five times and smoothed with spectropolarimeter system software, version 1.00 (Jasco, Japan). All measurements were performed under a nitrogen flow. The results are expressed in terms of molar ellipticity $(\theta)$. Measurements were carried out at $23^{\circ} \mathrm{C}$.

\section{Results and discussion}

To evaluate the stability of TMBP the conformational changes of TMBP induced by GdnHCl were studied (Fig. 1, left panel). Investigations were done by intrinsic protein fluorescence and far- and nearUV CD spectra. Fluorescence intensity at $320 \mathrm{~nm}$ remains practically unchanged in the range of $\mathrm{GdnHCl}$ concentration from 0 to $3.8 \mathrm{~mol} / \mathrm{l}$ (Fig. 1A). Than it dramatically decreases with $\mathrm{GdnHCl}$ concentration increase to $4.5 \mathrm{~mol} / \mathrm{l}$. The further increase of $\mathrm{GdnHCl}$ concentration does not influence on the fluorescence intensity. The dependencies of ellipticity at $222 \mathrm{~nm}$ and fluorescence intensity at $320 \mathrm{~nm}$ upon $\mathrm{GdnHCl}$ concentration are symbate (Fig. 1A). The parametric dependence between fluorescence intensities recorded on two slopes of fluorescence $\operatorname{spectrum}\left(I_{320}\right.$ and $\left.I_{365}\right)$ fits linear approximation (Fig. 1B). 

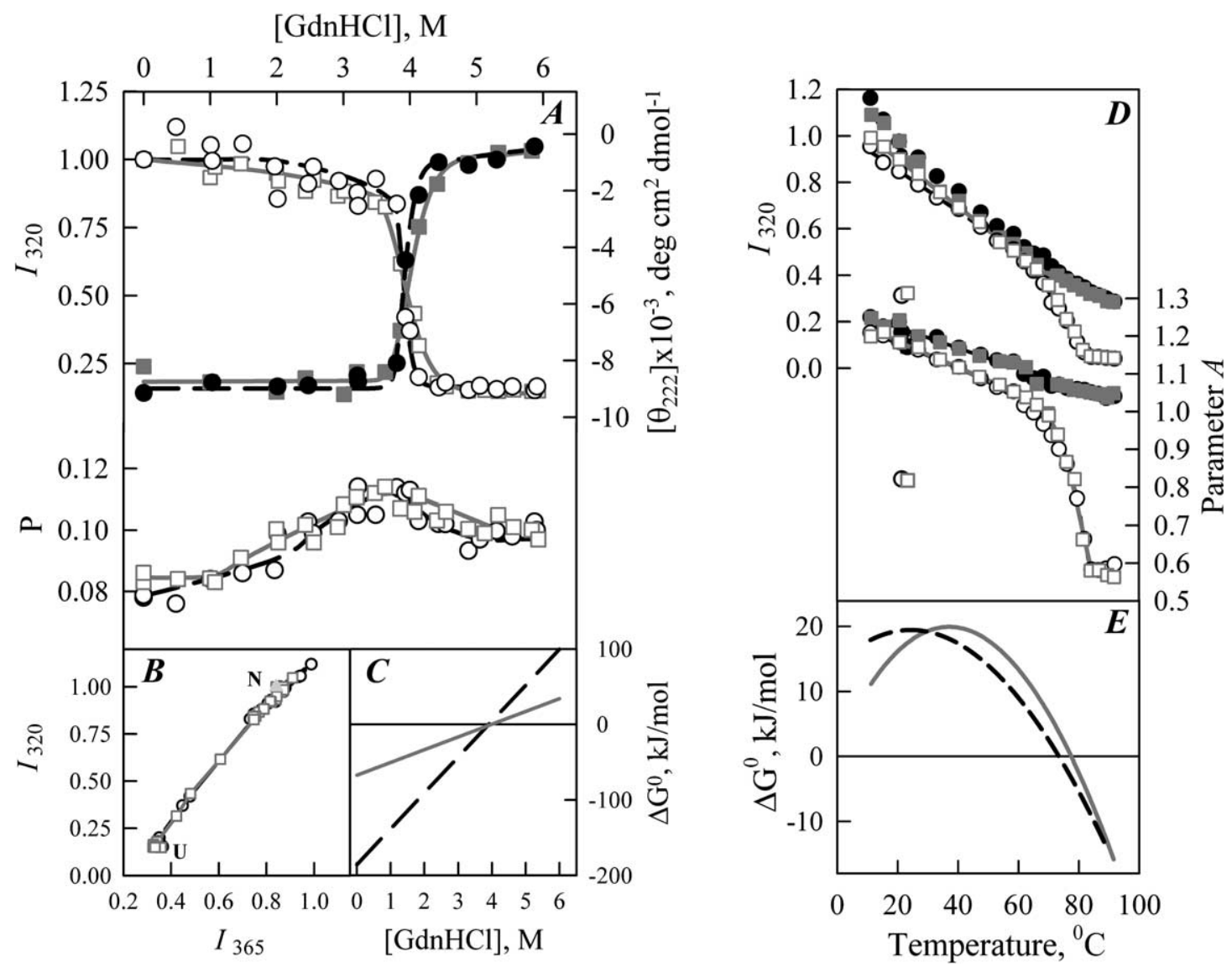

Fig. 1. GdnHCl-induced (left panel) and thermal (right panel) denaturation of TMBP (black circles) and TMBP/Glc (gray squares). (A) Fluorescence intensity $I_{320}$ (opened symbols), ellipticity $\theta_{222}$ (closed symbols) and fluorescence polarization of TMBP and TMBP/Glc recorded after $24 \mathrm{~h}$ of incubation in the definite concentration of $\mathrm{GdnHCl}$. (B) Parametric relationships between $I_{320}$ and $I_{365} \mathrm{~nm}$, characterizing unfolding of TMBP and TMBP/Glc. (C) Calculated value of change of free energy of TMBP and TMBP/Glc at GdnHCl-induced unfolding. (D) Change in fluorescence intensity $I_{320}$ and parameter $A$ during thermal denaturation of TMBP and TMBP/Glc. Closed symbols correspond to change of fluorescence characteristics of TMBP and TMBP/Glc in the absence of $\mathrm{GdnHCl}$, opened symbols - in the presence of $2.8 \mathrm{M} \mathrm{GdnHCl}$. (E) Stability of TMBP and $\mathrm{TMBP} / \mathrm{Glc}$ in the presence of $2.8 \mathrm{M} \mathrm{GdnHCl}$ at various temperatures.

In detail principles of this method described elsewhere [5]. Good approximation of parametric dependence between fluorescence intensities recorded at two wavelengths and sigmoid character of spectral characteristics upon $\mathrm{GdnHCl}$ concentration allowed to conclude that TMBP unfolding is one stage process.

At the same time the registration of TMBP fluorescence polarization dependence upon $\mathrm{GdnHCl}$ seems to indicate the existence of some intermediate state which fluorescence polarization is higher than that for native and unfolded protein (Fig. 1A). It is necessary to mention that in native state TMBP has abnormally low value fluorescence polarization $(P=0.08)$. For total 409 residues TMBP contains as much as 12 tryptophan residues that is also not typical. In principle the low value of the fluorescence polarization can be explained by high intermolecular mobility and/or by the existence of the effective non-radiative energy transfer between tryptophan residues. Analysis of the microenvironment of all tryptophan residues revealed that mostly tightly packed are tryptophan residues $\operatorname{Trp} 257$ and $\operatorname{Trp} 295$, located 
not far from the sugar binding center. Mostly exposed to the solvent is Trp 168. Interestingly, three of tryptophan residues have " $t$ " conformation (Trp 203, Trp 261 and Trp 310). As it was shown earlier, tryptophan residues in such conformation are mostly unstrained [9]. The freedom of high frequency mobility, which can lead for low value of fluorescence polarization are mostly probable for tryptophan residues in such conformation. Thus the contribution of high intermolecular mobility in the determination of low fluorescence polarization could not be excluded. Nonetheless, as the value of fluorescence polarization of native TMBP is even lower than that of unfolded state of this protein in $6 \mathrm{~mol} / \mathrm{l} \mathrm{GdnHCl}$ (see Fig. 1A) we can conclude that mainly it is determined by high probability of non-radiative energy transfer between practically all tryptophan residues of this protein. The addition of $\mathrm{GdnHCl}$ in the range of 1-3.8 mol/l causes no changes in the 3D structure of protein that is seen in Fig. 1A. At the same time specific interaction of $\mathrm{GdnHCl}$ with protein can provoke local small changes revealed only which disturb energy transfer that lead to the increase of fluorescence polarization. The further increase of $\mathrm{GdnHCl}$ concentration $(3.8-6 \mathrm{~mol} / \mathrm{l} \mathrm{GdnHCl})$ leads to protein unfolding recorded by other parameters and accomplished by the decrease of fluorescence polarization.

The midpoint of transition from native to unfolded state for TMBP and its complex TMBP/Glc in the assumption that this transition is one stage process equals to 3.91 and $3.98 \mathrm{~mol} / \mathrm{l}$ of $\mathrm{GdnHCl}$ for TMBP and TMBP/Glc, respectively. We have evaluated the difference of free energy between native and unfolded state $\Delta G$ for ligand-free and ligand-bound protein (Fig. 1C). For both of them it appeared to be several time large than that of protein from mesophilic organism. Denaturation of TMBP induces by $\mathrm{GdnHCl}$ is irreversible process. When the concentration of $\mathrm{GdnHCl}$ is diminished from 5 to $1 \mathrm{~mol} / \mathrm{l}$ of $\mathrm{GdnHCl}$ the intensity of the protein recovers only for $75 \%$, parameter $A$ practically does not change, while the value of fluorescence anisotropy increases becoming twice large than that for native protein. At the same time renaturation from $2 \mathrm{~mol} / \mathrm{l} \mathrm{GdnHCl}$ is completely reversible (data not shown).

As would be expected the study of heat denaturation of TMBP and its complex with Glc shows that protein is not completely unfolded even at $95^{\circ}$ (Fig. 1, right panel). So for calculating Gibbs free energy when protein denaturation was induced by heating we used protein solution with different concentration of $\mathrm{GdnHCl}$. The calculations were performed according to the standard scheme [6]. The obtained data show that the structure of complex TMBP/Glc is more stable than that of TMBP. Thus $T_{\mathrm{m}}$ for TMBP and TMBP/Glc in $2.8 \mathrm{~mol} / \mathrm{l} \mathrm{GdnHCl}$ is 73.4 and $77.7^{\circ}$, respectively (Fig. 1E). Notably that TMBP is more stable than TMBP/Glc complex at temperature below $30^{\circ} \mathrm{C}$. This fact corresponds data obtained at GdnHCl-induced denaturation of TMBP and TMBP/Glc study.

\section{Conclusion}

The evaluation of difference between free energy of native and unfolded state for TMBP and TMBP/Glc showed that both of them are several times higher than that of proteins from mesophilic organisms. This property makes TMBP a good candidate as a sensitive element in biosensor systems for sugar control.

\section{Acknowledgements}

This work was supported by Grants NATO (CLG.983088) and MK-1181.2010.4, Program "MCB RAS”, Contracts with FASI (02.512.11.2277) and FAO (P1198). 


\section{References}

[1] L. De Stefano, A. Vitale, I. Rea, M. Staiano, L. Rotiroti, T. Labella, I. Rendina, V. Aurilia, M. Rossi and S. D’Auria, Enzymes and proteins from extremophiles as hyperstable probes in nanotechnology: the use of D-trehalose/D-maltosebinding protein from the hyperthermophilic archaeon Thermococcus litoralis for sugars monitoring, Extremophiles 12 (2008), 69-73.

[2] J. Diez, K. Diederichs, G. Greller, R. Horlacher, W. Boos and W. Welte, The crystal structure of a liganded trehalose/maltose-binding protein from the hyperthermophilic archaeon Thermococcus litoralis at $1.85 \mathrm{~A}, \mathrm{~J}$. Mol. Biol. 305 (2001), 905-915.

[3] P. Herman, I. Barvik Jr., M. Staiano, A. Vitale, J. Vecer, M. Rossi and S. D'Auria, Temperature modulates binding specificity and affinity of the D-trehalose/D-maltose binding protein from the hyperthermophilic archaeon Thermococcus litoralis, Biochim. Biophys. Acta 1774 (2007), 540-544.

[4] P. Herman, M. Staiano, A. Marabotti, A. Varriale, A. Scire, F. Tanfani, J. Vecer, M. Rossi and S. D'Auria, D-trehalose/ D-maltose binding protein from the hyperthermophilic archaeon Thermococcus litoralis: the binding of trehalose and maltose results in different conformational states, Proteins 63 (2006), 754-767.

[5] I.M. Kuznetsova, K.K. Turoverov and V.N. Uversky, Use of the phase diagram method to analyze the protein unfoldingrefolding reactions: fishing out the "invisible" intermediates, J. Proteome Res. 3 (2004), 485-494.

[6] B. Nolting, Protein Folding Kinetics. Biophysical Methods, Springer, Berlin, 1999.

[7] K.K. Turoverov, A.G. Biktashev, A.V. Dorofeiuk and I.M. Kuznetsova, A complex of apparatus and programs for the measurement of spectral, polarization and kinetic characteristics of fluorescence in solution, Tsitologiia 40 (1998), 806817

[8] K.K. Turoverov and I.M. Kuznetsova, Intrinsic fluorescence of actin, J. Fluoresc. 13 (2003), 41-57.

[9] K.K. Turoverov, I.M. Kuznetsova and V.N. Zaitsev, The environment of the tryptophan residue in Pseudomonas aeruginosa azurin and its fluorescence properties, Biophys. Chem. 23 (1985), 79-89.

[10] K.B. Xavier, L.O. Martins, R. Peist, M. Kossmann, W. Boos and H. Santos, High-affinity maltose/trehalose transport system in the hyperthermophilic archaeon Thermococcus litoralis, J. Bacteriol. 178 (1996), 4773-4777. 


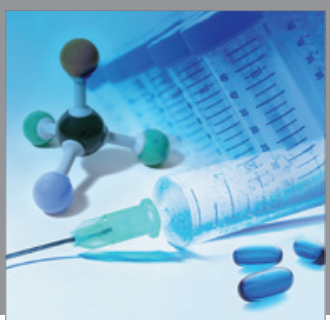

International Journal of

Medicinal Chemistry

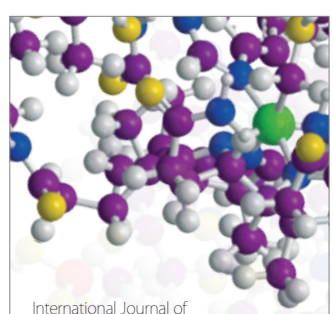

Carbohydrate Chemistry

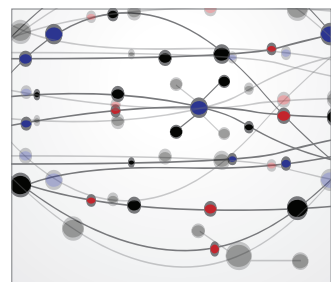

The Scientific World Journal
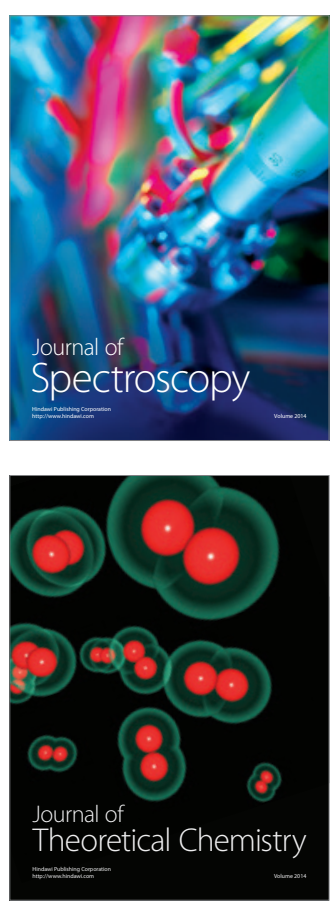
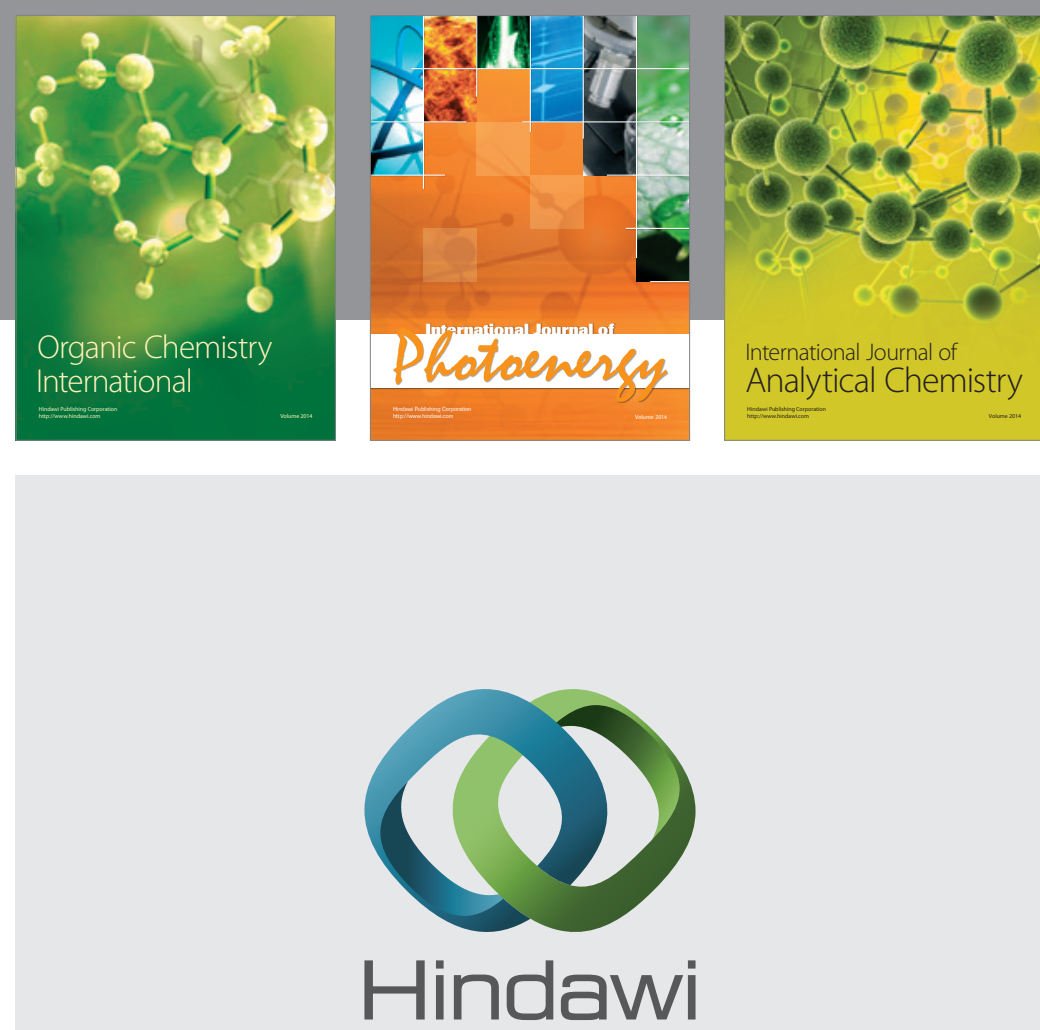

Submit your manuscripts at

http://www.hindawi.com
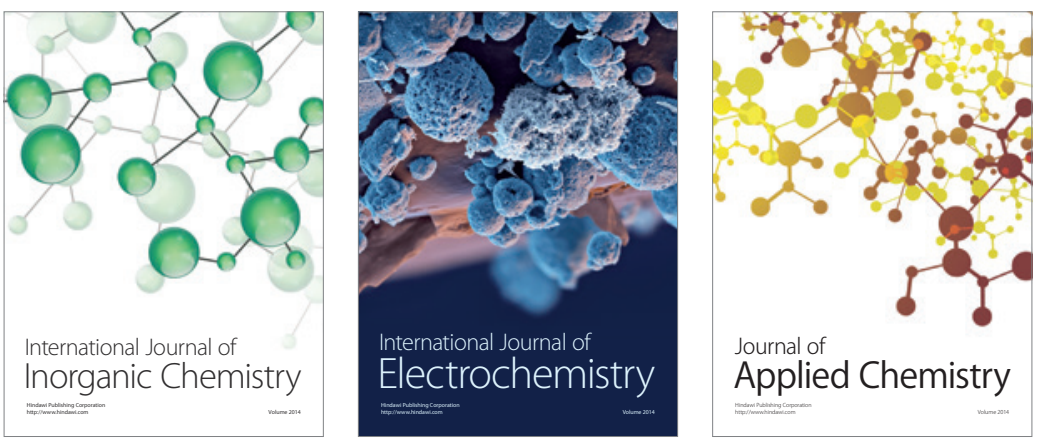

Journal of

Applied Chemistry
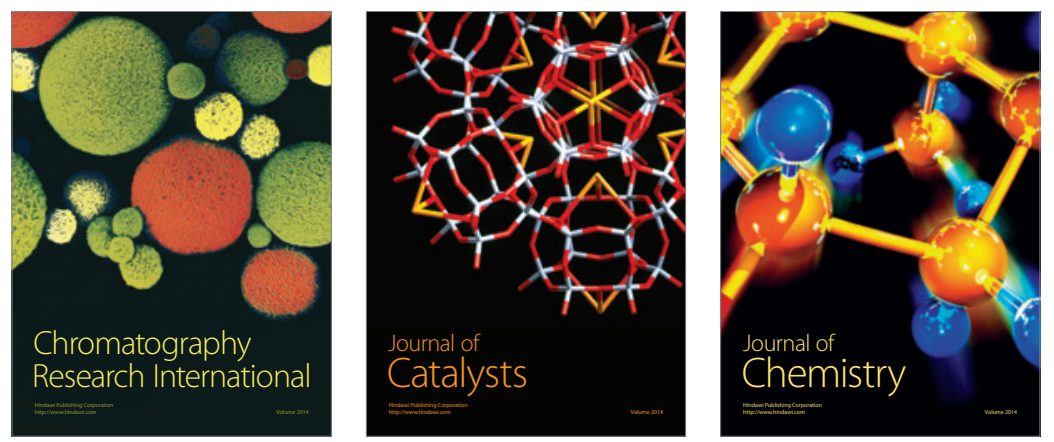
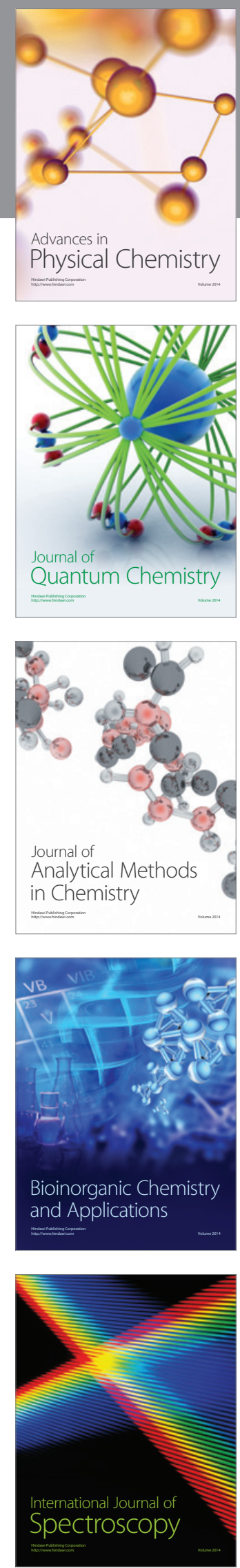